Revista de Filosofía

ISSN: 0034-8244

http://dx.doi.org/10.5209/RESF.62033

\title{
Ventajas y tensiones en la perspectiva del Estructuralismo Empirista
}

\author{
Bruno Borge ${ }^{1}$; Susana Lucero ${ }^{2}$
}

Recibido: 3 de abril de 2017 / Aceptado: 8 de junio de 2017

Resumen. En el presente trabajo analizamos críticamente el modo en que el Estructuralismo Empirista de van Fraassen caracteriza la relación de representación entre las teorías y los fenómenos. Nuestro objetivo es ofrecer argumentos que destaquen el papel del objeto en la construcción de modelos de datos. Asimismo, nos proponemos mostrar que la opción metodológica sugerida en su obra reciente resulta insuficiente para recuperar un vínculo plausible entre los modelos de datos y los fenómenos.

Palabras clave: estructuralismo empirista; empirismo constructivo; representación científica; modelos; van Fraassen.

\section{[en] Advantages and tensions of Empiricist Structuralism's perspective}

\begin{abstract}
In this paper we critically analyze how the relation of representation between theories and phenomena is characterized in van Fraassen's Empiricist Structuralism. Our aim is to offer arguments that emphasize the role of the object in building data models. We also propose to show that the methodological option suggested in his recent work is insufficient to recover a plausible link between data models and phenomena.
\end{abstract}

Keywords: empiricist structuralism; constructive empiricism; scientific representation; models; van Fraassen.

Sumario: 1. Introducción. Las tesis del Estructuralismo Empirista; 2. Los desafíos del Estructuralismo Empirista; 2.1. Realismo Estructural y Estructuralismo Empirista; 2.2. El problema del estructuralismo; 2.3. El problema de la relación de las teorías con el mundo; 2.4. Un análisis crítico del argumento de la indexicalidad; 3. Fenómenos, estructura y representación; 4. La metodología al rescate; 5. Conclusiones; 6. Referencias bibliográficas.

Cómo citar: Borge, B.; Lucero, S. (2018): "Ventajas y tensiones en la perspectiva del Estructuralismo Empirista", en Revista de Filosofía 43 (2), 315-338.

\footnotetext{
1 Universidad de Buenos Aires / IIF-CONICET

brunojborge@gmail.com

2 Universidad Nacional de Luján

susanalucero78@gmail.com
} 


\section{Introducción. Las tesis del Estructuralismo Empirista}

Bas van Fraassen, posiblemente el más destacado representante del anti-realismo científico de la actualidad, ha profundizado en sus últimas obras las nociones de representación y estructura con minucioso detalle. Estas ideas no estaban ausentes en la exposición de la Imagen Científica (1980), allí se hacía explícito un compromiso con la concepción semántica de las teorías y una noción de representación ligada al isomorfismo estructural. Asimismo, y dadas esas premisas, no era difícil concluir que la totalidad del conocimiento científico estaba signada por esa relación y que la idea de estructura se tornaría entonces preponderante. Sin embargo, no es sino hasta la primera década de este siglo cuando van Fraassen imprime a su filosofía un giro que la orienta de lleno hacia el estructuralismo científico. Esta maniobra ocurre en un marco general en el que la aparición y desarrollo del Realismo Estructural (Worrall, 1989; Ladyman, 1998) marcaron un nuevo rumbo en los debates clásicos en torno al realismo científico. Sin embargo, el estructuralismo de van Fraassen no lo conduce a un alejamiento de la visión antirrealista y empirista que definió su pensamiento desde el comienzo, pues continúa defendiendo la idea de que las ciencias empíricas son el paradigma de la investigación racional y que el objetivo de la ciencia es producir teorías empíricamente adecuadas y no globalmente verdaderas, en particular continúa enfatizando su rechazo a las demandas de explicación que conducirían la investigación hacia el terreno de la metafísica. (van Fraassen 2008, p. $3)$. El enfoque estructuralista de la filosofía vanfrassiana cristaliza en la formulación del Estructuralismo Empirista (EE), posición que conserva los mismos principios del Empirismo Constructivo pero incorpora varios elementos novedosos. En esta etapa dirige la atención con mayor intensidad hacia ciertos tópicos como la función de la representación en ciencia y emprende la tarea de revisitar y reformular cuestiones que habían sido abordadas con menor profundidad, como el problema de la referencia, la verdad, la indexicalidad y otros conceptos del análisis del lenguaje, todo lo cual contribuye sin duda a enriquecer su visión de la ciencia.

En vistas al objetivo del presente artículo, el problema de la representación y su rol en la producción y validación del conocimiento científico ocupan el tema central. Van Fraassen aborda este problema al describir la relación que se establece entre los modelos de una teoría científica y los fenómenos que ella investiga. Esta relación es mediada por la construcción de un modelo de datos que surge de las mediciones operadas sobre el fenómeno. Las mediciones adquieren, de este modo, una función muy importante, pues constituyen "el principal acceso inicial de la ciencia a los fenómenos" (2008, p. 87). En relación con los modelos de datos, van Fraassen introduce una interesante distinción entre fenómenos y apariencias que es de fundamental importancia para los argumentos con que fundamenta el rol de la representación. Los fenómenos se identifican con el evento observable; las apariencias, en cambio, son el fenómeno tal como es constituido por el usuario. La relación entre modelo de datos (apariencias) y fenómenos no es una simple relación diádica entre representación y evento representado, sino más bien una interrelación de tres lugares que incluye al usuario con sus específicos interese teóricos, objetivos epistémicos, instrumentos, etc., quien realiza las operaciones de observación y medición en un contexto. Es precisamente la actividad del usuario lo que hace posible que un modelo de datos sea la representación relevante de determinado fenómeno. (2008, p. 256) 
Señalemos en primer lugar que la tradicional dicotomía teoría-fenómeno es reemplazada por una distinción tripartita: teorías - apariencias - fenómenos donde las apariencias (contenido de las mediciones) actúan como las mediadoras entre la teoría y los fenómenos.

En segundo término, van Fraassen se aboca a priorizar la pragmática de la representación -lo que denomina la movida wittgensteiniana (2008, p. 254)-por sobre otros aspectos que se han destacado en la literatura corriente, como la similaridad entre el vehículo de la representación y su target. El representar debe entenderse fundamentalmente como una actividad, un proceso que comienza con las decisiones, preferencias e intereses de un agente sobre la base de ciertos compromisos teóricos previamente asumidos, y culmina con la elaboración de un modelo de datos que se construye a partir de los fenómenos y que será la piedra de toque para la contrastación de la teoría. En el comienzo de su tratado $(2008$, p. 23) van Fraassen deja sentado lo que denomina el Hauptsatz de su perspectiva acerca de la representación:

No hay representación excepto en el sentido de que algunas cosas son usadas, hechas o interpretadas para representar otras cosas de tal o cual manera.

Es por esa razón que opta por darle un "puesto de honor" (2008, p. 25) a la noción de uso; el énfasis está puesto en la pragmática, por sobre la sintaxis o la semántica de la representación en general" (2008, p. 25). En virtud de estos rasgos, la concepción de van Fraassen permite su inclusión dentro de lo que se conoce como el enfoque deflacionario acerca de la representación. ${ }^{3}$

Arribamos ahora al tercer componente novedoso que el EE aporta al análisis del estatus epistémico de las teorías y que resulta central para las consideraciones críticas que nos proponemos brindar en este trabajo: ¿en qué sentido la ciencia adopta el estructuralismo en el marco de una concepción empirista? La respuesta es que la ciencia produce un conocimiento de la estructura de los fenómenos, entendiendo que la estructura no es una propiedad intrínseca de los fenómenos. Más bien es la actividad humana de representarlos la que proyecta sobre ellos una estructura a fin de incluirlos en estructuras más amplias, los modelos teóricos. En este sentido puede decirse que la ciencia conoce la estructura de los fenómenos (van Fraassen 2006b). Esta tesis se plasma además en un rechazo de toda forma de realismo metafísico entendido, en este caso particular, como la afirmación de que el mundo tiene ciertas relaciones naturales o privilegiadas, una estructura objetiva independiente de nuestros intereses epistémicos.

Tales desarrollos motivaron una buena cantidad de análisis críticos que señalaron algunos puntos problemáticos del EE, vinculados principalmente a su caracterización de la relación entre la teoría y el mundo fenoménico. Si bien no fue presentado como

3 En la literatura contemporánea se ha instalado una taxonomía de las visiones de la representación que las clasifica en teorías sustantivas y deflacionarias. Las primeras definen la representación como una relación robusta entre dos términos: el vehículo (fuente) y la entidad representada (target), postulando condiciones necesarias y suficientes para que A represente B. Las teorías deflacionarias, por el contrario, ven la representación como una relación tripartita formada por un agente que usa un objeto (físico o abstracto) para representar algo de cierta manera, siguiendo las prácticas en uso en el contexto de una determinada área. El enfoque deflacionario asume también que la fuente suele permitir al agente informado realizar algunas inferencias en conexión con la fuente y el target. (Chakravartty 2010, Suárez 2015). 
una respuesta destinada a subsanar dichos aspectos conflictivos, un reciente trabajo de van Fraassen (2014) provee algunas consideraciones metodológicas, agrupadas en el Criterio de Fundamentación Empírica (Criterion of Empirical Grounding, de aquí en más $\mathrm{CEG}$ ), tendientes a clarificar el modo en que se gana un conocimiento objetivo acerca de los fenómenos mediante mediciones adecuadamente coordinadas con la teoría.

En el presente trabajo nos proponemos someter a crítica el modo en que van Fraassen caracteriza la relación entre las teorías, los modelos de datos que operan como representación de lo observable y los fenómenos mismos. En la segunda sección exponemos las principales tesis del EE y presentamos algunos desafíos que debe enfrentar esta concepción con vistas a ofrecer una fundamentación apropiada de la relación teoría - apariencias - fenómeno. Analizamos, en particular, al argumento de la indexicalidad y sus consecuencias, que derivan en lo que hemos denominado el problema del conocimiento objetivo y el problema de las restricciones a la representación, que son presentados y discutidos en la sección tercera. Nuestra conclusión es que el argumento es pertinente en el esquema general acerca del rol de la representación, pero no resulta eficaz como respuesta a la crítica central que se le ha formulado, una crítica que el propio van Fraassen bautizó como la objeción de pérdida de la realidad (2008, p. 258). En la cuarta sección, evaluamos la función promisoria del CEG y proponemos que debería complementarse con un criterio extra-metodológico que garantice el anclaje empírico de la teoría, un rasgo imprescindible en cualquier visión empirista de la ciencia. La quinta sección incluye las conclusiones y comentarios finales de este trabajo.

\section{Los desafíos del Estructuralismo Empirista}

\subsection{Realismo Estructural y Estructuralismo Empirista}

E1 EE, definido anteriormente en sus líneas generales, se presenta como un intento de desarrollar y completar las teorías elaboradas durante el siglo pasado por autores que suscriben el enfoque estructural en sus variadas modalidades. De acuerdo con el relato de van Fraassen, la creciente matematización de la ciencia que tuvo su comienzo en el siglo XVII culminó, en el siglo XX, con un giro estructuralista. Esta reorientación dio lugar a la aparición y desarrollo de variadas formulaciones del llamado realismo estructural, una concepción intermedia entre el realismo científico y el instrumentalismo (van Fraassen 2006b, p. 276). Van Fraassen ha expresado su rechazo de estas teorías debido a sus compromisos metafísicos y declara que "el estructuralismo encuentra su articulación apropiada solamente dentro de un planteo empirista" (van Fraassen 2008, p 237). De todos modos, para cualquier concepción estructural de la ciencia, la noción de representación es un concepto clave, entendida esencialmente como una estructura matemática. Una nota fundamental que diferencia las versiones del realismo estructural con respecto al EE es cómo debe entenderse el slogan "todo lo que conocemos es la estructura". Algunos partidarios del realismo estructural (Worrall 1989) afirman que la ciencia conoce la estructura del mundo que subyace en la realidad observable. En la versión de Worrall el realismo estructural sostiene que en el cambio científico hay acumulación de conocimientos; esta 
acumulación es mucho más que el contenido empírico de las teorías pero menos que el contenido teórico total. Hay continuidad o acumulación de la forma o estructura, no del contenido. (Worrall 1989, p. 157). Por su parte, los partidarios del realismo estructural óntico defienden la osada tesis de que conocemos la estructura de los hechos porque es lo único que hay (Ladyman 1998). Para un empirista, en cambio, la ciencia conoce la estructura de los fenómenos empíricos incluyéndolos en otras estructuras abstractas más amplias y estas solo pueden describirse -como señaló Weyl-hasta el límite del isomorfismo (2008, p. 238). Van Fraassen sostiene además que el EE es una visión acerca de las teorías científicas y no del mundo en el cual nos movemos. Ese mundo, al que hace referencia la ciencia, es el mundo plasmado en el contenido de sus modelos, lo que da sentido a la tesis de que lo que conocemos a través de la ciencia es la estructura. Si bien la demarcación vanfrasseana entre estas dos dimensiones del mundo es pertinente, parece necesario establecer un puente que las ponga en conexión, pues de otro modo la ciencia caería en riesgo de convertirse en un mero juego regido por reglas sin anclaje en la realidad observable en la cual todos nos movemos. Van Fraassen asume seriamente esta amenaza y proporciona un argumento elaborado (el argumento basado en la indexicalidad) a fin de resolver $-\mathrm{y}$ aun disolver-- el problema. A la vista de estas consideraciones, el EE se ve compelido a enfrentar algunos desafíos que su autor admite: una definición acerca del papel que desempeñan las estructuras en una teoría científica y el problema central de establecer una conexión plausible entre la teoría y el mundo (2008, p. 246); estas dos cuestiones se hallan íntimamente relacionadas. Debe notarse además que la nueva visión de la ciencia, al igual que el Empirismo Constructivo, postula la adecuación empírica como objetivo final de las teorías; de modo que la resolución de los dos problemas mencionados debe articularse apropiadamente con esta meta general.

\subsection{El problema del estructuralismo}

El problema del estructuralismo es presentado en forma de una pregunta que cuenta con lejanos antecedentes históricos (Russell, Carnap, Reichenbach, citados por van Fraassen 2008, p. 239 y ss.): ¿cómo es que una estructura abstracta, por ejemplo un modelo matemático, puede representar un fenómeno físico que, por definición, no es una entidad abstracta? Una respuesta ingenua a la pregunta es una clase de maniobra que van Fraassen denomina el gambito realista y que resuelve el problema rápidamente: el fenómeno de que se trata ya está naturalmente estructurado y la ciencia construye una copia- representación, tal que el fenómeno "encaja" en aquélla (van Fraassen 2008, pp. 240 y ss). Pero esta respuesta ingenua está preñada de supuestos metafísicos, a saber: (i) las estructuras están dadas en la naturaleza y los fenómenos instancian alguna estructura; (ii) la relación entre la representación y el fenómeno físico corre paralela a la idea de verdad como correspondencia, una relación de adecuación entre las expresiones del lenguaje y sus referentes extralingüísticos. (2008, p. 244)

Respecto del primer presupuesto, un empirista genuino rechaza el compromiso con la existencia de estructuras en el mundo:

Para el empirista constructivo, las teorías no se refieren al mundo. Más bien se refieren al mundo como es descripto por nosotros. Esas descripciones, sin embargo, no nos dicen nada acerca de la estructura real del mundo -si es que tal cosa existe-porque el hecho de 
que atribuyamos estructura a la naturaleza no implica que haya alguna base en el mundo que garantice esa atribución (van Fraassen 2006b, p. 158)

Aceptar la autonomía de las estructuras lleva consigo comprometerse con la existencia de universales, hacedores de verdad y otras nociones metafísicas reñidas con el empirismo. En realidad, no conocemos nada acerca de la estructura real del mundo. Más bien, la estructura es algo que le atribuimos a la naturaleza cada vez que la describimos:

Luego, ¿conocemos algo acerca de la estructura real del mundo? No, si lo que queremos decir es que la estructura es una entidad, algo así como un Universal, una reificación de lo que se predica. [...]Por otra parte, si saber que hay 9 planetas en órbitas alrededor de nuestro sol cuenta como un caso de conocer la estructura del mundo, entonces sí, la conocemos. (van Frassen 2006b, p. 158).

En lo que respecta al segundo presupuesto, la mirada realista de la representación está emparentada con la teoría de la verdad como correspondencia, un concepto que van Fraassen parece haber abandonado en los escritos recientes, a favor de una visión pragmática deflacionaria. Este tema se retomará en la siguiente sección. ${ }^{4}$

La pregunta reformulada es entonces ¿cómo es que un fenómeno, que carece de estructura como propiedad intrínseca, es capaz de ser incluido en un modelo matemático? Van Fraassen responde que las estructuras fenoménicas proyectadas por el usuario de una representación cristalizan en un modelo de datos (que también es una estructura matemática), con el objeto de ser incluidas (embbeded) en los modelos teóricos. Y puesto que la inclusión es de una estructura matemática en otra estructura matemática más amplia, no hay ninguna dificultad en relacionarlas vía isomorfismo, homomorfismo o lo que sea. Ahora podemos decir en un sentido comprensible que la ciencia "conoce" la estructura de los fenómenos, siempre y cuando la afirmación no implique una reificación de nuestras categorías cognoscitivas.

\subsection{El problema de la relación de las teorías con el mundo}

El segundo problema acerca de la conexión entre la teoría y los fenómenos es central con respecto al objetivo general de adecuación empírica. Recordemos que una teoría es empíricamente adecuada si los modelos que representan los fenómenos de su dominio mantienen una relación de isomorfismo estructural con los fenómenos. (2008, p. 249). La pregunta pertinente es ¿cómo, o en qué sentido, tal entidad abstracta como un modelo puede "salvar" o fracasar en "salvar" este fenómeno concreto? (2008, p. 245). La respuesta es que la vinculación entre la teoría y el fenómeno se opera mediante un modelo de datos que es también una estructura matemática. Si el fenómeno es empíricamente accesible, el modelo de datos construido por el usuario representa el fenómeno, pues es el resultado de observaciones y mediciones que efectivamente se tomaron bajo la orientación de teorías. Cuando se cumple la relación de isomorfismo estructural entre partes relevantes seleccionadas del modelo

4 Cabe recordar que van Fraassen hizo un mea culpa por haber usado anteriormente (La Imagen Cientifica) la noción de verdad en los términos característicos de la vision correspondentista. (Van Fraassen 2006b, p. 153. Respuesta a Rosenhagen). 
teórico y el modelo de datos, la teoría ha "salvado" el fenómeno (es empíricamente adecuada). En este punto surge la objeción que interpone el realista: el modelo teórico no se articula con el fenómeno real sino con un modelo de datos construido por el usuario. En rigor, la teoría no salva los fenómenos, salva las apariencias. Pero como el mismo van Fraassen señala, haciendo hablar al realista: "inosotros queremos que salve el fenómeno! (2008, p. 256).

A fin de resolver el problema, van Fraassen desarrolla el argumento de la indexicalidad. Toda la cuestión es abordada por el filósofo dentro de un marco esencialmente pragmático. El concepto clave es la idea de indexicalidad, una noción que entra en juego típicamente en algunas expresiones del lenguaje donde figuran palabras como aquí, allá, para nosotros, etc. Estas expresiones refieren al contexto en el cual se profieren los enunciados o juicios indexicales. El mismo fenómeno aparece además en toda clase de representaciones. Sean pictóricas, diagramáticas o abstractas, las representaciones requieren que el usuario se auto adscriba en el espacio lógico de la representación. Van Fraassen recurre a la comparación con los mapas, que son considerados en un plano de igualdad con los modelos (2008, p. 77). Frente al mapa de una región, el usuario tiene que fijar un punto que indica "yo estoy aquí"; ésta es la condición de posibilidad para el uso del mapa. La auto-adscripción no está contenida en el mapa, es una información extra necesaria para su uso. Estas mismas consideraciones se aplican a los modelos o representaciones científicas del fenómeno en estudio.

Volvamos al desafío de pérdida de la realidad. En conformidad con la tesis de auto- adscripción en el modelo, resulta central la relación del usuario con la representación y su articulación con la teoría. Cae de suyo, por tanto, que en un contexto en el cual un determinado modelo es una representación del fenómeno construida por el usuario, no hay diferencia, para esa persona, entre la cuestión de si la teoría se articula con su representación y la cuestión de si la teoría se articula con el fenómeno. (2008, p. 260). Estas dos cuestiones son lo mismo para el usuario; al declarar esta equivalencia, el usuario formula una tautología pragmática, esto es, un enunciado lógicamente contingente pero que es innegable para quien lo profiere (2008, pp. 259-260). De este modo, la teoría ha entrado en contacto con la realidad fenoménica. Van Fraassen afirma que comprender que la equivalencia para nosotros es una tautología pragmática disuelve la objeción de Pérdida de la Realidad. (2008, p. 259).

El argumento de la indexicalidad ha sido objeto de varias críticas encaradas desde diferentes perspectivas. En su artículo "Indexical Truth and Antimetaphysical Inclinations", Rosenhagen (2006) realiza un escrutinio de las nociones de verdad que figuran en textos de van Fraassen correspondientes a períodos distantes en el tiempo. El autor detecta dos conceptos de verdad: en La Imagen Científica la verdad es concebida en sentido correspondentista, como una relación entre las teorías científicas y el mundo. En esa etapa van Fraassen admite que existe un mundo causal y conceptualmente independiente de la mente (2006, p. 85). La postura coincide con el concepto de verdad que aplican los realistas científicos, la diferencia estriba solamente en el alcance. Sin embargo, en sus escritos recientes, van Fraassen introduce una versión idiosincrática de verdad: las teorías entran en relación con el mundo como es descripto por nosotros. No se habla de objetos, eventos o propiedades reales sino de descripciones o apariencias. La verdad de una proposición es tácitamente indexical de nuestro lenguaje (2006, p. 88). De acuerdo con Rosenhagen, estas dos nociones de 
verdad son incompatibles. Si la verdad tácitamente indexical se aplicara a la propiedad de observable, resultaría que ya no puede ser construida realísticamente, sería una pieza de teoría, nada más. Y lo mismo sucede con respecto a la afirmación de que "existe un mundo causal y conceptualmente independiente de la mente". Este último postulado se vuelve incomprensible. Dado que algunos constructivistas, idealistas e inclusive pragmatistas lo han negado, no es una afirmación tácitamente indexical (vale decir, un truismo de nuestro lenguaje, algo que no puede ser consistentemente negado), sino que es simplemente falso (2006, p. 90). Rosenhagen concluye su artículo con una recomendación:

Para permanecer en línea con sus inclinaciones empiristas anti-metafísicas y con su reciente deflacionismo, sugerimos que sería mejor que van Fraassen abandonara esos últimos resabios de realismo, que deje de confiar en la idea de un mundo independiente de la mente y que se una al idealismo o al pragmatismo. (Rosenhagen 2006, p. 91).

Por su parte, J. Nguyen (2016) denuncia que detrás del argumento de la equivalencia pragmática subyace el supuesto según el cual los actos de representación ocasionan compromisos doxásticos. Nguyen demuestra que ese supuesto es falso (2016, p. 182). Así, un científico que utilice un modelo para representar un fenómeno físico no contrae, por ese acto, ningún compromiso epistemológico con respecto a la exactitud de su representación o con la ubicación del target en el espacio lógico del modelo. Aun así, puede derivar predicciones acerca del target usando el modelo. El autor observa que van Fraassen da por sentado que el modelo de datos que ha construido el agente es un modelo preciso o exacto (accurate), pero no ha resuelto una cuestión previa, la de saber cómo es posible la representación en general (simpliciter), más allá de su eventual precisión. En otras palabras, el usuario puede creer o descreer que su modelo representa con precisión el fenómeno investigado, o que simplemente lo representa, sin embargo su creencia (o descreencia) no está implicada por los compromisos pragmáticos del acto de representar. "Hay una diferencia pragmática vital entre los actos de representación y los actos de aserción", es decir los primeros no contraen los mismos compromisos pragmáticos que los segundos. (2016, p. 183). Un ejemplo que ilustra la situación es el siguiente: un artista podría dibujar una caricatura de Margaret Tatcher como draconiana y, al mismo tiempo, permanecer agnóstico acerca de los rasgos de carácter de la ex primer ministro británica. Hipotéticamente, otro caricaturista, que fuera devoto del partido conservador y hubiera aceptado realizar el trabajo a pedido del partido laborista, pintaría a M. Tatcher como draconiana, a pesar de descreer completamente que la dama haya tenido los rasgos que muestra la caricatura (2016, p. 185). Luego, el argumento de van Fraassen a favor de la equivalencia pragmática ha fallado. Nguyen concluye que, a menos que van Fraassen esté dispuesto a reconsiderar la idea de que las estructuras deben buscarse "en el mundo", le quedan dos opciones: o bien renunciar a ofrecer una concepción estructural de la representación, o bien asumir una visión radicalmente anti-realista. Si esto último, su concepción de la ciencia sería implausible, pues tal declaración equivale a afirmar que las teorías no representan fenómenos o eventos observables sino modelos, es decir objetos matemáticos abstractos que son el producto de nuestra actividad intelectual independiente (2016, p. 189).

Los comentarios críticos elaborados por Nguyen se aproximan a las conclusiones a las que había arribado Rosenhagen; ambos autores coinciden en enfatizar las 
tensiones que se generan entre algunas consecuencias derivadas del análisis de la representación y ciertas tesis centrales de la filosofía de van Fraassen, como el realismo acerca de los objetos cotidianos, la noción de observabilidad o los aspectos pragmático-epistémicos de la representación. Los dos artículos concluyen con recomendaciones referidas a abandonar posiciones muy arraigadas en la filosofía de van Fraassen y adoptar formas extremas de anti realismo. Por nuestra parte, desarrollamos argumentos críticos que van en la misma dirección, arriesgando la hipótesis de que los pilares que sostienen el empirismo constructivo y el estructuralismo empirista reclaman ser revisitados en conformidad con el enfoque estructural de sus obras recientes.

\subsection{Un análisis crítico del argumento de la indexicalidad}

Nuestros comentarios acerca del problema de la indexicalidad siguen dos líneas de argumentación: (i) Una arista de nuestra crítica es acerca de la pertinencia del argumento indexical como respuesta a la objeción de pérdida de la realidad. Demostramos que la respuesta de van Fraassen es inapropiada debido a que no se atiene al contexto en que es formulada la pregunta del realista metafísico. (ii) En segundo término mostramos que la tesis de la equivalencia pragmática se funda, en gran parte, en las analogías trazadas por van Fraassen entre la representación científica y el carácter representacional del lenguaje. Las nociones clave a las que apela corresponden a conceptos semánticos como denotación, referencia y verdad. Ofrecemos razones para sostener que esas analogías tienen a los sumo una función heurística, pero son insuficientes para fundamentar la tesis.

Comenzaremos con el segundo tópico (ii). Van Fraassen declara que la objeción interpuesta por el crítico contiene implícitamente una noción de verdad como correspondencia, en el sentido en que es entendida por la semántica abstracta, es decir la disciplina que explora la relación entre los signos lingüísticos y las cosas. $(2008,252)$. Son centrales en esta área las nociones de referencia, denotación y verdad, pero en la medida en que no se vinculen con la práctica, es decir con el uso, los usuarios y el contexto, esas nociones no tienen una significación relevante en el lenguaje. Estamos en pleno derecho de buscar una interpretación de los conceptos semánticos y esta interpretación se encuentra en la pragmática del lenguaje: (2006b, p. 154). La denotación, por ejemplo, cumple el rol referencial que se le asigna sólo si se hace patente su contrapartida pragmática. Así, la oración "el término W denota el objeto O" es una abreviatura de la oración "la persona x usa el término W para referirse al objeto $\mathrm{O}$, en el momento t". En el primer enunciado, los parámetros contextuales se han abstraído o quedan fijos pero es posible explicitarlos; de allí que en su formulación abstracta, la oración es tácitamente indexical. Lo mismo cabe decir respecto de la verdad. La oración "S es verdadera" tiene como contrapartida pragmática "la persona $X$ hace una afirmación verdadera de la situación $\beta$, mediante el uso de la oración $\mathrm{S}$ en el momento t". En general, las aseveraciones relativas a términos de la semántica como los mencionados son típicamente tautologías pragmáticas. (2006b, p. 156). Van Fraassen agrega que muchas veces se han ignorado las abstracciones que opera la semántica del lenguaje, lo que ha conducido a algunos filósofos desprevenidos a entrar en dilemas metafísicos. (2006b, p. 156).

Las elucidaciones de los conceptos semánticos que anteceden reciben especial atención en los escritos más recientes de van Fraassen, lo que parece revelar un 
cambio drástico de opinión en los temas de interés a lo largo de los años (2006b, pp. 153-154). La importancia otorgada a las cuestiones lingüísticas manifiesta, en nuestra opinión, una revalorización del papel del lenguaje en ciencia, especialmente por su estrecha relación con las características de la representación, "el lenguaje es el asiento del significado, si es que algo lo es" (2008, p. 84). Esta declaración da pie para concebir la representación del mismo modo como se abordan los conceptos de referencia y verdad. En otras palabras, la representación no puede concebirse separadamente de la práctica de representar. Así, lo que determina que un modelo sea la representación de un fenómeno no es una relación independiente de toda otra cosa entre el vehículo y el target; el modelo representa en virtud de la acción de un agente que actúa en un contexto disciplinar determinado. Entendemos que el hecho de traer al escenario del debate los análisis del lenguaje le permite a van Fraassen no solamente construir una concepción pragmática de la representación científica, sino también fundamentar el argumento indexical con el cual pretende eliminar la objeción de pérdida de la realidad.

Además el carácter indexical de la verdad también afecta la idea de adecuación empírica, lo que ha sido objeto de severas críticas, como ya lo hemos señalado. Van Fraassen reformula el concepto en los siguientes términos: "Las teorías empíricamente adecuadas son verdaderas en un sentido tácitamente indexical. Ellas nos dicen cosas - usando nuestro propio lenguaje- que no podemos negar consistentemente" (2006b, p. 158). Creemos que detrás de esta caracterización subyace el supuesto de que hay dos tipos de hechos cuya comparación es aproblemática (el lenguaje y la representación), de modo que los análisis efectuados en uno de estos planos, por ejemplo en el lenguaje, pueden proyectarse, sin mayores problemas, al terreno de la ciencia. En el presente trabajo cuestionamos el supuesto y consecuentemente la pretensión de haber disuelto el problema de la relación de la teoría con el mundo.

Notamos que las analogías entre la representación científica y el lenguaje son problemáticas, pues en principio debería demostrarse que el lenguaje es un tipo de representación. En este punto es oportuno traer al debate las objeciones formuladas por M. Ghins (2010). Para Ghins, las oraciones que refieren a hechos no son representaciones de esos hechos y tampoco la inversa, las representaciones no son proposiciones. "Afirmo -y soy conciente de que esto es bastante controversial-que el lenguaje no representa el mundo, sino que lo describe, correcta o incorrectamente". (Ghins 2010, p. 527. Las cursivas pertenecen al autor). Por el momento podemos dejar de lado esta aguda observación y aceptar la premisa de que el lenguaje representa, en pro de la argumentación. En lo que sigue, vamos a atenernos al caso de la representación científica. Como sea, la relación entre las expresiones de un lenguaje natural y sus denotados parecen, a primera vista, tener un paralelo con la relación entre el vehículo de la representación -la fuente-y su target. En este especial aspecto, podemos decir que la fuente (el modelo científico) cumple una función denotativa.

El tema ha sido analizado en detalle por Mauricio Suárez (2010). Este autor introduce la noción de fuerza representacional de la fuente, como una propiedad más liberal que la denotación. La primera no requiere que el denotado sea una entidad real, la fuente puede referirse a un objeto ficcional como el flogisto, por eso es una noción apropiada para el uso en contextos científicos. Además la fuerza representacional de una fuente está validada por la práctica científica de representar en un área determinada, no es un mero acto de estipulación. De todos modos, en 
nuestra opinión, las analogías entre el lenguaje natural y la representación científica no van más allá de este rasgo. Tomamos como testigo el lenguaje natural porque los ejemplos de van Fraassen son del tipo "la nieve es blanca", "algunas manzanas son rojas", "la superficie de mi mesa es cuadrada". En tanto sistema de signos, el lenguaje natural es un producto histórico-social compartido por una comunidad de hablantes, ellos no lo han construido, aunque puedan modificarlo gradualmente. Van Fraassen estaría de acuerdo con esta idea porque así lo manifiesta en otro lugar. En "Replies to the papers" afirma:

¿Cuál es la marca de un "buen" lenguaje? Un lenguaje es el lenguaje de una comunidad, si es que, en realidad, es un lenguaje vivo; y es "bueno" si sirve bien a las prácticas de esa comunidad. Este criterio es totalmente empírico y pragmático. Nótese que no depende por completo de la comunidad que ésta tenga un buen lenguaje. En algún momento su lenguaje cesa de servir bien y necesita cambios; éstos sobrevienen inesperadamente, y ciertamente no por elección. (van Fraassen 2006b, p. 150).

Ahora bien, mediante el uso del lenguaje natural, los hablantes habitualmente no abrigan otro propósito más allá de la referencia misma. En algunos casos los hablantes asocian ciertas notas generales que componen la connotación de un término, en particular si el término es un nombre propio. Veamos ahora si el uso de la representación en ciencia es homologable a la pragmática del lenguaje. De acuerdo con la concepción de van Fraassen, el objetivo de la ciencia es ofrecer teorías empíricamente adecuadas. La ciencia persigue este objetivo mediante la construcción de modelos destinados a articularse con la estructura de los fenómenos, los modelos de datos. Para ello debe elaborar un complejo montaje que incluye mediciones y observaciones, sistemas de medición, teorías observacionales y de los instrumentos, acuerdos y decisiones metodológicas, todo ello orientado por los intereses del investigador y en concordancia con la teoría que determina la relevancia de esas operaciones.

Otra vez los argumentos de Ghins resultan muy atinentes: la relación de representación tiene lugar entre estructuras, pero el fenómeno bajo estudio no tiene ninguna estructura. Es preciso construir la estructura fenoménica, extraerla del objeto por abstracción y luego producir un homomorfismo entre el modelo de datos y la estructura fenoménica que es "parasitaria del fenómeno o hecho real" (Ghins, 2010) y agregaríamos: el modelo de datos no representa el fenómeno real sino la estructura del fenómeno; es -por decirlo así- una construcción de segundo grado, la construcción de primer grado corresponde a la construcción de la estructura fenoménica. Ciertamente el modelo de datos está construido en un sentido en que las palabras de nuestro lenguaje no lo están.

En efecto, ninguno de los complejos factores mencionados más arriba está involucrado en la relación de los signos lingüísticos y sus referentes. Por supuesto que en la representación científica como en el lenguaje, las condiciones de contexto están siempre presentes, sea en forma tácita o explícita y, en tal sentido, se podría asimilar la intencionalidad de uso de la representación a la contrapartida pragmática de la semántica, a la que alude van Fraassen; sin embargo, las diferencias de contexto son tan marcadas que el paralelo no justifica ni podría justificar el argumento indexical.

En primer lugar, los modelos científicos son estructuras matemáticas comparables sólo hasta el límite del isomorfismo. Esta es una diferencia esencial con respecto a 
la referencia de los términos y oraciones del lenguaje natural. En segundo lugar, la participación activa de los usuarios en la construcción y uso de los modelos teóricos es primordial para la actividad científica, o simplemente coincide con ella. Por el contrario, la comunidad de hablantes ha recibido el legado del lenguaje como parte de la tradición y la cultura del mundo en el que viven y que no pueden modifica a voluntad. En palabras de van Fraassen "[...] en algún momento su lenguaje [el de la comunidad] cesa de servir bien y necesita cambios; estos sobrevienen inesperadamente y, ciertamente, no por elección". (van Fraassen 2006b, p. 150. Las cursivas son nuestras). En tercer lugar, los científicos construyen modelos para representar los fenómenos en pos de lograr objetivos esencialmente epistémicos (formular predicciones). Recordemos que las teorías científicas son artefactos construidos para ayudarnos a planificar y comprender (2008, p. 238). En cambio, el lenguaje natural es usado con múltiples propósitos $\mathrm{y}$, en algunos contextos, los objetivos que se persiguen son definitivamente extraños a los intereses de conocimiento.

Concluimos que la fundamentación del argumento indexical, basada en las comparaciones de la representación científica con los conceptos lingüísticos, no cumple el rol que se le atribuye; sobre todo debido a las significativas diferencias, especialmente pragmáticas, que observamos en los dos campos de aplicación. En este sentido la crítica de M. Ghins (2016) puede aportar una razón valedera: los modelos y los enunciados pertenecen a diferentes categorías de $\operatorname{cosas}^{5}$. Aun cuando las analogías entre el lenguaje y la representación científica puedan aportar intuiciones esclarecedoras, tienen una función heurística, nada más. Por sí mismas, no alcanzan a fundamentar el argumento indexical que disolvería - tal lo afirmado por van Fraassen-la objeción de la pérdida de la realidad.

Pasemos entonces al problema (i) acerca de la pertinencia del argumento indexical. Van Fraassen utiliza el ejemplo de un científico que ofrece un gráfico para representar el crecimiento de la población de ciervos en Princeton. Su gráfico ha sido construido a partir de mediciones y una serie de operaciones ejecutadas de acuerdo con las prácticas vigentes. Una teoría $\mathrm{T}$ provee un modelo que se articula muy bien con la estructura desplegada en la representación gráfica. (2008, p. 254). Entre la audiencia, un crítico formula una pregunta. Esta persona no cuestiona la corrección de la representación, su inquietud se refiere a si el modelo teórico está representando efectivamente el fenómeno real, la población de ciervos, y no una estructura abstracta construida por el científico. Posiblemente tenga en mente el carácter no transitivo de la representación: si el modelo teórico representa un modelo de datos y éste, a su vez, representa un fenómeno observable, de allí no se sigue que el modelo teórico representa el fenómeno. Acerca de este hipotético cuestionamiento, van Fraassen explica que uno no puede presentar un gráfico como representando algo y al mismo tiempo decir que tal vez no lo represente en absoluto. Menciona la paradoja de Moore: "esto es así pero yo creo que no lo es". Afirmar los dos conyuntos sería incurrir en una contradicción pragmática, es decir un enunciado lógicamente contingente pero que no puede negarse. (2008, p. 260). Para el usuario,

\footnotetext{
En su artículo "Bas van Fraassen on Success and Adequacy in Representing and Modelling”, M. Ghins defiende la idea de que denotar y representar son dos acciones diferentes. La denotación es una condición necesaria para la representación, pero no es una condición suficiente. Además de denotar, el usuario asume creencias acerca de la fuente (representator) y acerca del target, sin las cuales no podría elaborar las estructuras correspondientes; y esas creencias se formulan en enunciados. Un enunciado no es una representación. (Ghins 2016, pp. 30-31, las cursivas nos pertenecen).
} 
su modelo y el fenómeno son lo mismo; si negara la equivalencia, incurriría en la paradoja de Moore, es decir, caería en una contradicción pragmática.

Desde nuestro punto de vista, el cuestionamiento del crítico ha recibido una respuesta satisfactoria en el orden de lo pragmático. En efecto, el científico tiene que localizar el fenómeno en el espacio lógico del modelo, del mismo modo que el usuario de un mapa tiene que fijar un punto del mapa que indica "yo estoy aquí", a fin de ubicarse en la región representada (el objeto real) y poder recorrerla. En cualquier caso, lo que determina todo el proceso es el "uso" de un objeto (su estructura) para acceder al target mentado y esta relación se expresa en juicios indexicales (2008, p. 258).

La solución propuesta, a nuestro criterio, no pone punto final a la historia. En primer lugar, es necesario desbrozar dos cuestiones que aparecen entrelazadas en el hipotético diálogo entre el científico y su interlocutor: el problema de la corrección o exactitud de la representación, la cual --aun si fuera incorrecta no dejaría por eso de ser una representación-- $\left(\right.$ Suárez, 2010) ${ }^{6} \mathrm{y}$, por otra parte, el hecho de que la teoría representa efectivamente el fenómeno. La pregunta del oponente se refiere al segundo punto. Como ya hemos visto, la respuesta de van Fraassen es mostrar la equivalencia pragmática del usuario. La cuestión es que el crítico no está discutiendo las actitudes proposicionales del agente, ni dudando de que el científico incurra en inconsistencias pragmáticas. Con relación al contexto en que la pregunta es formulada, la tautología pragmática del usuario es irrelevante. El oponente apunta a algo diferente de las actitudes y creencias del usuario, pregunta por algo objetivo. Van Fraassen advierte que el crítico no está planteando una cuestión epistemológica y sospecha -a lo largo del diálogo-- cuál es su verdadera intención, algo del nivel de lo metafísico. (2008, p. 255).

Desde nuestro punto de vista, la objeción del crítico es acerca de la representación misma como producto de una actividad, y aquí es importante tener en mente que el hecho de que los parámetros pragmáticos -usuarios y contexto-sean inescindibles del acto de representar no impide realizar un escrutinio analítico que examine otros rasgos además de los pragmáticos. ¿Por qué un examen de la representación no puede dejar fijos los parámetros contextuales y focalizar en las relaciones de representación de la teoría con respecto al fenómeno? Van Fraassen ha expuesto claramente la diferencia que hay entre la contingencia lógica de una afirmación, que corresponde al contenido de un enunciado, y la negabilidad o asertabilidad que son conceptos pertenecientes al uso, son actos de un usuario del lenguaje. (2008, p. 260). Se sigue entonces que si el juicio es indexical o contextualmente dependiente, la respuesta pragmática es la adecuada. Por nuestra parte notamos que, en este caso particular, la objeción del interlocutor es acerca del contenido de la afirmación sobre la relación Teoría-Fenómeno, de allí que la respuesta resulte finalmente inapropiada de cara a la perspectiva en que se ha formulado la pregunta. La objeción del interlocutor se orienta a un aspecto de la representación científica y el argumento indexical responde desde otro. En suma, la pregunta es legítima y tenemos derecho a formularla independientemente de nuestras lealtades filosóficas. La presunción de

Mauricio Suárez sostiene que con frecuencia los modelos científicos son inexactos, sin embargo no pierden, por eso, su capacidad de representar. "Un modelo M imperfecto e inexacto acerca de un sistema target T, es todavía un modelo de T". [...] "Los problemas relativos a la noción de representación son previos e independientes de las cuestiones sobre la exactitud" (Suárez 2010, p. 93). 
que el oponente lleva consigo una latente preocupación metafísica no parece exenta de algún prejuicio. Por cierto, la visión pragmática ha enriquecido enormemente nuestra comprensión de cómo se lleva a cabo una representación en ciencia pero no ha resuelto el problema de fondo: la conexión de la teoría con el mundo.

Para concluir, el enfoque pragmático que impregna toda la visión vanfrasseana de la representación pone el énfasis en el uso que gobierna la práctica científica y el uso implica invariablemente el accionar de un sujeto (o de un grupo) en situación. A este respecto, Van Fraassen admite la posibilidad de que se haya dejado entrar la subjetividad en la ciencia y aclara que esta amenaza queda excluida porque se funda en un error (2008, p. 83). La ciencia conserva un "residuo ineliminable de subjetivismo" que se refleja en la necesidad del usuario de auto-adscribirse al espacio lógico del modelo, es decir en la referencia a la situación empírica particular que protagoniza el agente. Si la indexicalidad esencial implica conciencia y agentes, entonces tienen que ser incluidos porque están involucrados en la premisa del uso (2008 p 83). De todos modos, esa circunstancia no compromete la objetividad. Citando a Hermann Weyl, recuerda que debemos admitir un residuo de "aniquilación del ego", que permanece aun en las ciencias más teóricas (2008, p. 87).

Nuestro punto de vista es que la cuota de subjetivismo -entendida en un sentido no peyorativo, como afirma van Fraassen- es inocua y constituye una condición de posibilidad para la construcción y el uso de modelos. Agreguemos además que es necesario tener presente la "no aniquilación" del objeto. Por cierto, es igualmente importante atender a los rasgos no construidos del objeto, a los que se les ha concedido escaso papel en el esquema general de la representación. Según van Fraassen cómo sea el fenómeno tomado en sí mismo no determina qué estructuras son sus modelos de datos, eso depende de nuestras decisiones al atender a ciertos aspectos del fenómeno, a representarlo de ciertas maneras y hasta cierto punto. (2008, p.254). Sin embargo hay un sentido en que el fenómeno tomado en sí mismo condiciona, en cierta medida, el accionar del usuario; lo hace bajo la forma de poner límites a la clase de modelos que se pueden construir. Para tomar un ejemplo muy a mano en la literatura corriente, la caricatura de Margaret Tatcher, dibujada por un artista que la presenta con rasgos distorsionados, no deja lugar a dudas de que representa a Margaret Tatcher y no a la reina Isabel Segunda. Si ocurriera esto último, entonces la caricatura no la representa.

La naturaleza de los fenómenos observables - con sus disposiciones propias no construidas ni ensambladas-- es el target final para la adecuación empírica de una teoría. Al igual que el accionar del sujeto, el evento observable y sus rasgos específicos son la otra condición necesaria limitante de los modelos que la ciencia puede construir para representarlo. A menos que se admita esta premisa, el empirismo de van Fraassen se coloca al borde del constructivismo en su versión fuerte, una forma radical de antirrealismo (como sugiere Nguyen) ${ }^{7}$. Si la naturaleza cumple un papel central en el EE, necesitamos saber cuál es y de qué manera intervienen los fenómenos (no las apariencias) como condición de posibilidad ontológica para la construcción de los modelos científicos.

\footnotetext{
La caracterización de Constructivismo Científico Fuerte se ajusta a la taxonomía de A. Kukla (2000). En la versión fuerte, el constructivismo científico postula la tesis según la cual los hechos científicos (en particular los hechos de las ciencias naturales), a los que podemos tener acceso, son construidos o inventados dentro de las instituciones de la ciencia (laboratorios, experimentos de campo, etc); éstos abarcan los hechos que actualmente conocemos y todos los que vayamos a conocer. (Kukla, A. 2000, pp. 24-25).
} 


\section{Fenómenos, estructura y representación}

Nuestras críticas al intento vanfraassiano de eludir la objeción de la pérdida de la realidad han tenido como objeto iluminar un aspecto problemático de la filosofía de van Fraassen, a saber, su caracterización de la relación entre la teoría y el mundo que ésta pretende representar. A este respecto la restricción de los compromisos epistémicos al plano de lo observable - es decir, al terreno de la mera adecuación empírica - no resulta suficiente para disipar las dudas respecto de cómo las subestructuras empíricas de la teoría brindan información acerca del mundo fenoménico. Las razones para esas dudas residen en la articulación de la doctrina vanfraassiana de la representación como actividad con el rechazo del realismo de las estructuras. Más allá de las dificultades asociadas a la objeción de la pérdida de la realidad, sostenemos que dos problemas adicionales socavan el planteo del EE. El primer problema deriva de la afirmación conjunta de dos compromisos que, por diversas razones, van Fraassen incorpora en el EE. Llamaremos a esta primera cuestión el problema del conocimiento objetivo. Para arribar al segundo problema, denominado aquí el problema de las restricciones de la representación, se requiere una estrategia ligeramente diferente. Sostenemos que existe una tensión general entre la adopción conjunta de los compromisos asumidos por el EE que solo puede resolverse abandonando al menos uno de ellos. El EE no puede tenerlo todo.

Comencemos por listar los compromisos que van Fraassen pretende incorporar a su EE

(0) Realismo del Sentido Común.

(1) Rechazo del realismo acerca de la estructura del mundo.

(2) Una concepción estructuralista del conocimiento científico.

(3) Una visión pragmática de la representación.

(4) Una distinción entre representaciones correctas e incorrectas.

(0) y (1) son herencia del Empirismo Constructivo. Es así como van Fraaseen afirma que "el Empirismo Constructivo se encuadra en un realismo del sentido común que fue ajeno a buena parte de la tradición empirista" (2002, p. 479). De hecho, el realismo del sentido común parece afirmase como un realismo directo, en conjunción con una teoría de la verdad como correspondencia respecto de los fenómenos: "podemos ver, y de hecho vemos la verdad acerca de muchas cosas: nosotros mismos, otros, árboles y animales, nubes y ríos, en la inmediatez de la experiencia" (van Fraassen 1989, p. 178).

Por su parte, (1) es la negación de la tesis que adjudica al mundo una estructura identificada con relaciones naturales, relevantes o privilegiadas. Esto, según van Fraassen, no compromete la adopción de (0):

Los metafísicos pueden afirmar que el realismo del sentido común es ininteligible excepto que se presuponga una base metafísica, codificada en la frase de Platón en el Fedro: 'se debe cortar la naturaleza por sus articulaciones' (...) Rechazo esa afirmación. Considero que el sentido común y su referencia no problemática a niños y toboganes es inteligible sin esa base. (2010, p. 548) 
Con respecto a los dos compromisos (0) - (1) dos puntos deben ser notados. Primero, la negación del realismo acerca de la estructura del mundo afecta incluso las entidades observables acerca de las cuales van Fraassen está dispuesto a adoptar un robusto realismo. Segundo, la actitud frente a esa tesis no es el agnosticismo, como ocurre en el caso del Realismo Científico, sino su completo rechazo. De hecho, van Fraassen dedica un notable esfuerzo a mostrar que la negación de una tesis metafísica no tiene por qué ser otra tesis metafísica (2009, p. 479)

Por su parte, (2) y (3) son incorporaciones que han recibido un agudo y pormenorizado tratamiento en el EE. Como hemos visto, ambos representan respectivamente una reacción al surgimiento del Realismo Estructural (2) y la declaración del Hauptsatz de su perspectiva acerca de la representación (3). En cambio, el compromiso enunciado en (4) no aparece bien clarificado en la obra del filósofo, a pesar de que se puede razonablemente juzgar esencial a cualquier concepción que quiera preservar el carácter objetivo del conocimiento científico; o como el mismo van Fraassen lo expresa, para "no aterrizar en el idealismo subjetivo" (van Fraassen 2006a p. 545). Se trata, después de todo, de la muy relevante diferencia entre representar exitosamente el mundo y fracasar en esa empresa, de dejar abierta la posibilidad de representar inadecuadamente un aspecto de la realidad.

Un primer aspecto conflictivo del problema del conocimiento objetivo, deviene de la afirmación conjunta de (1) y (2). En pocas palabras: ¿cómo es posible ganar conocimiento objetivo puramente estructural de algo que no tiene ninguna estructura objetiva? ${ }^{8}$ Una posible respuesta es que la negación del realismo acerca de la estructura del mundo no implica que los fenómenos carezcan de toda estructura, sino solo que ninguna estructura es privilegiada o naturalmente relevante. Sin embargo, esto no nos acerca siquiera a una solución. El problema, de hecho, es tan viejo como la objeción de Newman (1928) al realismo estructural de Russell (1927). Sumariamente, lo que ahora se conoce como el problema de Newman consiste en que dado un conjunto $C$ de cardinalidad $n$ es posible adjudicar a $C$ infinitas estructuras relacionales coherentes con $n$. De ese modo, la afirmación de que $C$ tiene alguna estructura desconocida es un conocimiento trivial que se sigue de la lógica y la cardinalidad de $C$. Una intuición semejante descansa detrás del célebre argumento modelo-teórico de Putnam. Van Fraassen es plenamente consciente de estas dificultades. Es por ello que su solución al problema del conocimiento objetivo no es otra que el argumento de la indexicalidad. Somos nosotros quienes a través de nuestras actividades de representación adjudicamos al mundo fenoménico una estructura, una entre las infinitas posibles. Y esa estructura no es relevante en sí misma, sino para nosotros.

[T] odo lo que realmente podemos decir es que hay una estructura en nuestras descripciones de aquello que queremos describir -nada nuevo se añade si sustituimos "aquello que queremos describir" por "mundo" o "naturaleza". Las divisiones marcadas en nuestras descripciones (...) son aquellas que resultan importantes para nosotros. Esta importancia se resume en su totalidad diciendo "ellas son nuestras" (van Fraassen 2009, p. 471)

Así, en virtud de la indexicalidad, no existe diferencia pragmática real entre decir que conocemos la estructura del mundo y afirmar que conocemos la estructura

Versiones de este problema han sido formuladas en Iranzo (2014) y Borge (2015). 
que atribuimos al mundo. Consideramos que dicha estrategia es fallida, y dado que nuestras consideraciones críticas acerca del argumento de la indexicalidad han sido expuestas en secciones anteriores, no nos detendremos más en este punto. Con todo, un segundo problema puede vislumbrase ya en este planteo. Si no hay diferencia en la práctica entre atribuir una estructura $a$ los fenómenos y conocer la estructura de los fenómenos, ¿cuál podría ser la base para sustentar una distinción entre representaciones adecuadas e inadecuadas? Esto nos conduce a lo que hemos llamado el problema de las restricciones a la representación. Una forma alternativa de dar con esta dificultad es considerar los compromisos listados más arriba. El EE afirma un realismo fuerte acerca de los fenómenos (es decir, de las entidades y procesos observables), sin embargo niega que corresponda a estos alguna estructura de relaciones natural o privilegiada. El EE insiste, además, en exaltar los componentes pragmáticos de la representación, dando un papel central a las decisiones del científico en la conformación de los modelos de datos que serán la piedra de toque para todo juicio de adecuación empírica de una teoría. Cada una de las mediaciones introducida por el proceso de representación entre los fenómenos y las subestructuras empíricas está, como ya hemos hecho notar, pragmáticamente determinada. Son los intereses, objetivos y estrategias particulares del científico las que condicionan tanto la construcción de un modelo de datos a partir de los fenómenos, como su adecuación respecto de la teoría que se pretende contrastar. Es así que:

No hay nada en una estructura abstracta en sí misma que pueda determinar que es el modelo de datos relevante para ser ajustado a la teoría. Un modelo de datos particular es relevante porque se construyó sobre la base de resultados recogidos de una cierta manera, seleccionados por criterios específicos de relevancia, en ciertas ocasiones, en un entorno experimental u observacional práctico, diseñado para tal fin. (van Fraassen 2008, p. 253)

Los modelos de datos son una representación puramente estructural de los fenómenos, pero estos no tienen una estructura objetiva, no hay en ellos relaciones relevantes o privilegiadas listas para ser representadas. Ahora bien, si no hay nada en una estructura abstracta que la determine como el modelo adecuado para representar cierto fenómeno y, por otra parte, el fenómeno en sí mismo carece de una estructura objetiva que pueda ser seleccionada como la interpretación privilegiada, todo el peso de la adecuación entre fenómeno y modelo de datos recae sobre el modo en que el científico construye las apariencias, es decir, sobre su decisión de representar el fenómeno de cierta manera y de acuerdo con ciertos intereses. No es fácil ver cómo este modo de caracterizar la relación de representación pueda evitar el siguiente resultado indeseable: no existen criterios objetivos para distinguir entre representaciones adecuadas e inadecuadas de los fenómenos. Este relato no muestra más que la tensión existente entre la afirmación de (0)-(3) por una parte y (4), por otra. La adopción de los primero parece minar la posibilidad de contar con una distinción sustantiva entre representaciones exitosas y no exitosas de los fenómenos. El defensor del EE, en suma, no puede tenerlo todo.

Hay, desde ya, más de una manera de resolver esa tensión. Algunas de ellas son, no obstante, inconvenientes a todas luces en este marco. El rechazo de (0), por ejemplo, implicaría apartarse de un aspecto esencial de la doctrina vanfraassiana desde el Empirismo Constructivo, y probablemente de los límites habituales del debate acerca del realismo científico. Del mismo modo, resignar (3), es decir, 
abandonar una perspectiva pragmática respecto de la representación implicaría sin más el abandono del principal aporte del EE, si no de la propia posición por completo. Como hemos visto, existen razones de peso compartidas por el mismo van Fraassen para no sacrificar (4). A este respecto, la analogía con las discusiones en torno al argumento modelo-teórico son nuevamente pertinentes. En el contexto de ese debate, Merrill (1980) consideró que el foco del impacto del argumento de Putman se encuentra en una caracterización incompleta de la tesis realista, puesto que ésta no debe incluir solo la creencia de que el mundo es nada más que una clase de entidades, sino además que "esas entidades mantienen unas con otras ciertas relaciones objetivas" (Merrill 1980, p. 72, la cursiva pertenece al original).

El movimiento más conveniente parece ser entonces rechazar (1), es decir, abstenerse de negar de plano el realismo acerca de la estructura. Una posición menos contundente sería abstenerse de afirmar o negar la existencia de estructuras objetivas en el mundo; tal actitud no está en absoluto reñida con la perspectiva pragmática de la representación. Así entonces, un proceso de representación exitoso tendrá como output una descripción adecuada, pero cuál sea esa descripción dependerá, sin dudas, de cuáles hayan sido los intereses y énfasis del agente en dicho proceso, y demás factores contextuales. La consecuencia de adoptar una postura como la que sugerimos, de prudente agnosticismo, no equivale a comprometerse con que exista una forma privilegiada de describir la estructura de los fenómenos independiente de todo factor contextual, sino más bien que --una vez fijado el contexto-- solo la atribución de ciertos rasgos estructurales (a saber, los que corresponden a algún rasgo de la estructura del mundo postulada hipotéticamente) resulta en una descripción adecuada.

En lo que atañe al problema de las restricciones a la representación, la solución de van Fraassen (2014) consiste, en esencia, ofrecer una fundamentación no metafísica de (4), es decir, sustentar una distinción entre representaciones adecuadas e inadecuadas con un criterio puramente empirista. Se trata de lo que denominó Criterion of Empirical Grounding (CEG), inspirado en la obra de Weyl (1927/1693). Buena parte de la motivación para este criterio está dada por el reconocimiento de la importancia de los procesos de medición en la construcción de los modelos y de su relación problemática con la teoría que obra de marco para dichos procesos. "[S] i un proceso es o no una medición, y si lo es, qué es lo que mide, son preguntas que tienen, en general, respuesta sólo en relación con una teoría". Sin embargo, el carácter teóricamente dependiente de esos procesos no debe arrojarnos a "temores escépticos", dado que dichas "respuestas, proporcionadas por la teoría, son parte de lo que permite a una teoría cumplir con el requisito estricto de fundamentación empírica [empirical grounding]'. (van Fraassen 2014, p. 81).

Si los temores escépticos pueden ser disipados es porque el carácter teóricamente dependiente de la medición no impide que los modelos de datos se refieran también a los fenómenos con los que debe contrastarse la teoría. Tal dependencia teórica incluye, sin dudas, todas las mediaciones pragmáticas involucradas en la construcción de modelos. Es así como el CEG podría brindar una respuesta satisfactoria a los problemas presentados en esta sección, sin contaminar el EE con compromisos metafísicos. En otras palabras, aceptar (4) sin tener que resignar (1). En la siguiente sección analizamos el carácter y la presunta eficacia del CEG. 


\section{La metodología al rescate}

El CEG pretende brindar una garantía de que la influencia de la teoría sobre los procesos de medición no sea tal que la construcción de modelos de datos acabe por volverse algo así como un juego interno, un diálogo de la teoría con sus propias expectativas divorciado del tribunal de la experiencia. Con todo, esa garantía no apela a compromisos metafísicos, sino que se mantiene fiel al espíritu empirista del EE. Si el CEG hace su trabajo, una distinción entre representaciones exitosas y fallidas sería posible. Los requisitos metodológicos que componen el CEG son presentados por van Fraassen en su (2014, p. 98). Algunos de ellos no sólo eran parte de la concepción de Weyl, sino son recomendaciones más o menos comunes a toda reflexión metodológica acerca de la práctica científica. Así, por ejemplo, el CEG prescribe que dado algún parámetro teóricamente relevante, éste debe poder ser medido. Del mismo modo, la aplicación de distintos sistemas de medición de un parámetro debe estar coordinada, de manera que haya concordancia en los valores determinados por diferentes medios. No emprenderemos aquí un análisis pormenorizado de cada una de las prescripciones de este criterio, nos concentraremos más bien en la función que tiene dentro del EE.

El CEG impone profundas restricciones al desarrollo de modelos de datos mediante la aplicación de reglas metodológicas precisas, tanto respecto de su relación con la teoría como de su anclaje en las apariencias. No cualquier modelo es adecuado para cualquier fenómeno. El problema del conocimiento objetivo y el problema de las restricciones a la representación quedarían así neutralizados.

Hay algunas razones para dudar de la contundencia de este resultado. El CEG puede limitar severamente el rango de modelos de datos adecuados para un fenómeno y asegurar que la 'contaminación teórica' de la medición no afecte su referencia objetiva, pero así y todo continúa siendo un problema cómo caracterizar una relación de representación epistémicamente relevante entre una entidad capaz de brindar un conocimiento puramente estructural (el modelo de datos), y algo que carece de toda estructura (el fenómeno). Ignoraremos aquí estas dificultades y supondremos que el CEG constituye una herramienta que es en principio capaz de lidiar con el problema del conocimiento objetivo. Nos abocaremos, en cambio, a responder dos preguntas concernientes a su lugar, función y eficacia en el marco del EE respecto del problema de las restricciones a la representación: primero, ¿qué es CEG? Segundo, ¿cuáles son las razones últimas para adoptarlo?

La primera de esas preguntas encuentra una respuesta sencilla. El CEG es una limitación metodológica, una "restricción normativa a la ciencia (....) respecto de qué cuenta como una medición, o como una medición de qué" (van Fraassen 2014, p. 97). El rumbo que toman los argumentos de van Fraassen puede anticiparse sin demasiado esfuerzo si se atiende a la evolución de su filosofía. El EE podría solucionar el problema de lograr un anclaje satisfactorio a la experiencia restringiendo el rango de modelos de datos adecuados para contrastar la teoría y asegurando una independencia relativa de los procesos de construcción de modelos respecto de los principios teóricos que los guían. Para lograr este objetivo, algunos realistas han modelado la metodología en torno a las nociones de confirmación/ corroboración. El éxito predictivo de la teoría se interpreta como un signo de su verdad probable o de su aproximación a la verdad. La acumulación de confirmaciones experimentales y la capacidad de anticipar nuevos tipos de fenómenos son incluso leídas como señales 
de la confiabilidad de las teorías de bajo nivel acerca de lo observable, aquellas que determinan en parte la construcción de modelos de datos. De ese modo las restricciones normativas del método procuran que los procesos involucrados en la contrastación de la teoría se orienten hacia su creciente acuerdo con los hechos que pretende describir. Pero esta estrategia, tomada en su totalidad, no sería un camino posible para el defensor del EE, pues su objetivo no es alcanzar la verdad aproximada o la confirmación de la teoría sino "salvar los fenómenos"; y para eso, cuenta con la metodología como recurso más eficaz. No obstante, el vínculo con la experiencia ha de mantenerse como uno de fundamentación (grounding). Van Fraassen reconoce esta situación sin reservas:

[el CEG] constituye una ruptura con la opinión de que el científico se ocupa de confirmar hipótesis teóricas, o de justificar creencias. A este respecto mi posición se asemeja a la de Frederick Suppes (1993), según la cual el científico se ocupa de credencializar en lugar de confirmar hipótesis, aunque no en su relación con el constructivismo social. (2014, p. 97)

Esta situación decanta en la segunda de las preguntas que orientan el desarrollo de esta sección: ¿qué justificación puede encontrar el defensor del EE para la adopción del CEG como estándar metodológico? Van Fraassen no va más allá de proponer su criterio en los términos ya mencionados, no ofrece ninguna justificación para ello. No es dificil, sin embargo, imaginar dos respuestas posibles a dicha pregunta: (i) no hay de hecho razones para brindar una justificación más allá del hecho de que el CEG forma parte de la práctica científica; (ii) la razón para su adopción reside en su eficacia para aumentar el grado de adecuación empírica de las teorías.

Consideremos primero (i), en una formulación más elaborada: no es necesario aventurarse en las oscuras aguas de la especulación metafísica, en la semántica o la epistemología para dar cuenta de la pertinencia del criterio. El CEG es parte de la práctica científica, impone a ésta tan sólo restricciones normativas. En tanto conjunto de reglas, puede ser justificado apelando simplemente a consideraciones pragmáticas y metodológicas, derivadas de convenciones inherentes a dicha práctica.

Esta primera respuesta esconde un error fundamental. Es cierto que la formulación de un criterio normativo para la práctica científica (que en tanto conjunto de reglas consiste solo en indicaciones de qué debe hacerse y qué debe evitarse) no involucra, en principio, ninguna tesis acerca de qué cosas existen, ni cómo o con qué medios se las conoce. Como es cierto también que las decisiones convencionales son comunes en la práctica científica, al punto en que muchas veces modelan dicha práctica y los criterios que la rigen. Sin embargo, la adopción de un criterio hace que éste herede de la práctica en la que se enmarca sus objetivos y razón de ser. Del mismo modo que el Empirismo Constructivo, el EE fija como meta para la ciencia y su práctica la adecuación empírica. Si bien en tanto objetivo es mucho más modesto que la verdad aproximada de las teorías, no deja de tratarse de un objetivo epistémico: la ciencia persigue "una continua acumulación de conocimiento empírico a lo largo del cambio teórico" (van Fraassen 2006a, p. 537). Así que, aun cuando sea correcto afirmar que el CEG no implica por sí compromisos semánticos, epistémicos o metafísicos, las razones para su adopción quedan supeditadas al objetivo epistémico. La práctica científica tendría que ser acorde con la meta propuesta, sin embargo las decisiones y reglas metodológicas, sin otro sustento que la propia práctica, se revelan insuficientes para justificar el objetivo de adecuación empírica. 
Por su parte, (ii) afirma precisamente que el CEG es la metodología más indicada para alcanzar la creciente adecuación empírica de nuestras teorías. Aunque orientada al objetivo epistémico correcto, esta estrategia resulta también inconveniente. Las razones de su fracaso dependen de cómo se entienda el término 'adecuación empírica' en ese marco. Si lo que allí significa 'adecuación empírica' es la mera relación de isomorfismo entre el modelo de datos y las subestructuras empíricas correspondientes de la teoría que se está contrastando, el CEG resulta tan deseable como cualquier estrategia ad hoc que permita construir el modelo de datos indicado para asegurar dicho isomorfismo. Si el objetivo es solo establecer una función entre dos estructuras matemáticas, cualquier estrategia que permita acomodar dichas estructuras en una relación de isomorfía resulta igualmente válida. Con todo, podría replicarse, no puede ser eso lo que se quiere expresar con 'adecuación empírica' en el contexto de esta respuesta, sino más bien el hecho de que la relación de isomorfismo entre ambas estructuras matemáticas se da en una circunstancia tal en la que una de ellas, el modelo de datos, se hace "transparente", generando así un vínculo entre la teoría y la experiencia. Es decir, las subestructuras empíricas de la teoría, por medio del modelo de datos, resultan adecuadas a algún aspecto de los fenómenos mismos, dando así lugar a una distinción entre representaciones adecuadas e inadecuadas. Pero la pregunta obligada es, ¿cómo es que CEG asegura que eso suceda?

Es imperioso recordar que los fenómenos, en sí mismos, no tienen estructura. Pero incluso si ello no fuera un problema o pudiese superarse con el argumento de la indexicalidad (o algún otro), la pregunta permanece: ¿por qué el CEG es el recurso más adecuado para garantizar la relación entre teoría y fenómeno? Ello no puede simplemente afirmarse. Hacerlo equivaldría a pretender que el CEG generase fundamentación empírica sólo por llamarse de ese modo. ¿Por qué, entonces, la concordancia de valores para una misma variable -determinados sobre la base de conexiones teóricamente postuladas- por medios experimentales diferentes es un criterio más adecuado a los fines epistémicos de la ciencia que, digamos, la determinación de dicho valor por una sola vía? Algunas réplicas pueden parecer naturales: la coordinación de ambas mediciones muestra que hay algo en el mundo que se corresponde con las relaciones teóricamente postuladas. O bien: el hecho de que un mismo valor sea arrojado por dos o más vías diferentes no puede ser sólo una coincidencia. Pero ninguna de estas posibilidades está disponible para el EE, ya que ellas hacen uso de la intuición del argumento del no-milagro, que indica que la mejor explicación del éxito predictivo de las teorías es su verdad aproximada. La pura metodología atada a un puñado de razones pragmáticas no es capaz de llenar la brecha que existe entre el modelo de datos y el fenómeno, entre una relación de isomorfismo entre dos estructuras abstractas y la representación adecuada de la realidad observable. El objetivo de la ciencia es epistémico, y la metodología es estéril si no se complementa con consideraciones extra-metodológicas que den cuenta del modo en que ciertas restricciones normativas derivan en un mayor conocimiento sobre los fenómenos.

La razón por la que la pura metodología no es suficiente para tender un puente entre modelo de datos y fenómeno se vincula estrechamente con aquella detrás del fracaso de la primera respuesta a nuestra pregunta acerca de la justificación del CEG. Un criterio normativo para la práctica científica podría sustentarse en meras decisiones metodológicas referentes a la práctica científica si nos situáramos en un marco estrictamente convencionalista. Si el juego de la ciencia fuese, usando una 
expresión de Lakatos (1978, p. 122), un juego como cualquier otro, la interacción entre su práctica y sus reglas (la metodología) no tendría por qué estar condicionada por presupuestos metafísicos, o por la satisfacción de metas epistémicas. Pero el escenario es bien diferente en el caso del EE. Su afirmación de un realismo del sentido común - que se ha mantenido incólume desde las primeras formulaciones del EC- y la elección de la adecuación empírica como objetivo de la ciencia determinan que la práctica científica está orientada hacia un fin epistémico concreto (i.e., un conocimiento creciente y acumulativo acerca de la estructura de los fenómenos), de este modo, la elección de una metodología es injustificada a menos que se muestre su eficacia con respecto a dicho fin.

El punto de quiebre entre este relato realista acerca de lo observable y la metodología que tiene como meta la adecuación empírica yace en el rechazo de la tesis del realismo estructural. Si ninguna estructura objetiva impone restricciones a nuestras representaciones de los fenómenos, algo más debe hacerlo. El candidato de van Fraassen es el CEG, pero el problema es que las restricciones que éste impone no pueden vincularse con la satisfacción del objetivo epistémico de ganar un conocimiento (estructural) acerca de los fenómenos. 'Credencialización' de hipótesis o 'fundamentación empírica' lucen más como expresiones de deseo que como nombres de recursos genuinos capaces de sustituir la confirmación realista.

Ahora bien, creemos que es relevante notar que en su rechazo del Realismo Metafísico, van Fraassen llega aún más lejos que en su eterna batalla contra realismo científico y lo que llamó "metafísica inflacionaria” (van Fraassen 1980, 73). La tesis realista acerca de la existencia de las entidades inobservables postuladas por nuestras mejores teorías no es rechazada en favor de su opuesta, el instrumentalismo, sino reemplazada por una actitud agnóstica. Van Fraassen admite incluso que, si bien su posición empirista incluye el nominalismo modal, en tanto doctrina acerca del objetivo de la ciencia el Empirismo Constructivo es independiente de la adopción de compromisos modales y es compatible con un agnosticismo modal (Monton y van Fraassen 2003). Su rechazo del realismo metafísico, como hemos visto, es categórico: involucra la afirmación de que el mundo carece de toda estructuración objetiva.

No discutiremos aquí sus razones para sostener esta tesis. Nos limitaremos en cambio a sugerir que una manera de superar el problema de la insuficiencia del CEG, a fin de restablecer la brecha entre la teoría y los fenómenos, es dar lugar a un fundamento de la distinción entre representaciones adecuadas e inadecuadas. Si admitimos que los fenómenos investigados por las teorías son un subconjunto de los objetos y procesos observables, entonces ese grano de realismo que van Fraassen está dispuesto a aceptar puede ser el hilo conductor que garantice el interjuego entre teorías, modelo de datos y fenómeno. Podríamos ser agnósticos respecto de cuál sea la estructura real de los fenómenos sin necesidad de convertirnos en ateos. En otras palabras, el agnosticismo que van Fraassen abraza hacia arriba -con respecto a la existencia de entidades y procesos inobservables- bien puede ser proyectado hacia abajo, no respecto de los fenómenos observables cuya existencia no admite dudas, sino con relación a algún patrón de comportamiento que no depende de nuestra mente, lenguaje o siquiera de nuestra existencia. 


\section{Conclusiones}

Hemos afirmado que la existencia de los factores pragmáticos en la representación es incuestionable. El objetivo de este trabajo no es de ninguna manera desconocer la importancia de estos factores, sin embargo el llevarlos a primer plano no debería opacar el rol del fenómeno real que aparece bastante empalidecido en el esquema de van Fraassen. Desde nuestro punto de vista, resulta imprescindible ponderar la participación de los propios hechos en todo el montaje experimental, pues sus rasgos y características condicionan hasta cierto punto la clase de modelos de datos que pueden construirse. Esto no implica desconocer que la articulación final de la teoría es con las apariencias, es decir con el fenómeno tal como es descripto por nosotros.

En tal sentido, hemos criticadoel argumento delaindexicalidad delarepresentación. Asimismo, presentamos dos problemas que surgen de los compromisos que el EE pretende incorporar a su posición. Sostenemos, además, que la solución propuesta por van Fraassen a dichos escollos (el CEG) resulta insuficiente. Por último, hemos esbozado una propuesta alternativa que incorpora en el EE una dosis mínima de realismo que, creemos, no pone en jaque la actitud empirista global de la posición defendida por van Fraassen. El postular la existencia de rasgos propios del evento real no nos compromete con la tesis metafísica de que hay una estructura privilegiada y una única forma correcta de representación. Nuestra propuesta es una postulación hipotética, no una reificación. La flexibilidad subrayada por van Fraassen, en cuanto a que la estructura relevante del fenómeno es construida por el usuario y viene diseñada desde alguna teoría, no es incompatible con la creencia de que el fenómeno aporta sus propios atributos. Más bien, esta circunstancia es la condición de posibilidad de que puedan construirse modelos matemáticos que lo representen, en concordancia con nuestros objetivos epistémicos y en las condiciones apropiadas del contexto. Describirlo de otro modo nos coloca al borde de fusionar la realidad (la ontología) con los ingredientes epistémicos y pragmáticos que concurren en la actividad de representar.

\section{Referencias bibliográficas}

Chakravartty, A. (2010). Truth and representation in science: Two inspirations from art. In Beyond mimesis and convention (pp. 33-50). Springer Netherlands.

Ghins, M. (2010). "Bas van Fraassen on Scientific Representation", Analysis Reviews, 70, $\mathrm{N}^{\circ} 3$, pp. 524-536.

Ghins, M. (2016). "Bas van Fraassen on Success and Adequacy in Representing and Modelling" In Magnani and G. Casadio (eds.). Model-Based Reasoning in Science and Technology, Studies in Applied Philosophy, Epistemology and Rational Ethics 27.

Hacking, I. (1979): "Imre Lakatos's Philosophy of Science", The British Journal for the Philosophy of Science, 30, No. 4, pp. 381-402

Iranzo, V. (2014): "Models and Phenomena: Bas van Fraassen's Empiricist Structuralism", In W. González (ed.), Bas van Fraassen's Approach to Representation and Models in Science, Springer Netherlands, pp. 63-76.

Lakatos, I. (1978): The methodology of scientific research programmes: Philosophical Papers. Vol. I (J. Worrall \& G. Currie, Eds.), Cambridge, Cambridge University Press. 
Ladyman, J. (1998): "What is structural realism?" Studies in History and Philosophy of Science, 29, pp. 409-424.

Merrill, G. H. (1980) “The model-theoretic argument against realism”. Philosophy of Science 47: 69-81.

Monton, B. y van Fraassen, B. C., (2003). "Constructive Empiricism and Modal Nominalism". The British Journal for the Philosophy of Science 54, pp. 405-422.

Newman, M.H.A. (1928). "Mr Russell's Causal Theory of Perception". Mind, 37, pp. 137148.

Nguyen, J. (2016). "On the pragmatic equivalence between representing data and phenomena". Philosophy of Science, 83(2), 171-191.

Rosenhagen, R. (2006): "Indexical Truth and Anti-Metaphysical Inclinations". In A. BergHildebrand y C. Suhm (eds.), Bas van Fraassen: The Fortunes of Empiricism. Münstersche Vorlesungen zur Philosophie, De Gruyter, Germany, pp. 81-91.

Russell, B. (1927) The Analysis of Matter, London: George Allen \& Unwin.

Suárez, M. (2010) “Scientific Representation”, Philosophy Compass 5 (1): pp. 91-101. Blackwell.

Suárez, M. (2015a) “Deflationary Representation, Inference, and Practice”, Studies in History and Philosophy of Science, 49, pp. 36-47.

Suppe, F. (1993): “Credentialling scientific claims”, Perspectives on Science, 1, pp. 153-203. van Fraassen, B. (1980). The Scientific Image. Oxford, Clarendon Press.

van Fraassen, B.C. (1989) Laws and Symmetry, Oxford: Clarendon Press.

van Fraassen, B. C. (2002). On McMullin's appreciation of realism concerning the sciences. Philosophy of Science, 70(3), pp. 479-492.

van Fraassen, B. C. (2006a): Representation: The problem for structuralism. Philosophy of Science, 73, 5, pp. 536-547.

van Fraassen, B. C. (2006b): "Replies to the papers". In A. Berg-Hildebrand y C. Suhm (eds.), Bas van Fraassen: The Fortunes of Empiricism. Münstersche Vorlesungen zur Philosophie, De Gruyter, Germany, pp. 125-171.

van Fraassen, B. C. (2008). Scientific Representation: Paradoxes of Perspective, Oxford, Oxford University Press.

van Fraassen, B. C. (2009): “Can Empiricism Leave Its Realism Behind? Toward a Dialogue with Trascendentalists". In M. Bitbol, P. Kerszberg and J. Petitot (eds.), Constituting Objectivity, Springer, Dordrecht, 459-480.

van Fraassen, B. C. (2010). "Reply to Contessa, Ghins and Healey", Analysis, 70, pp. 547556.

van Fraassen, B. C. (2014. "The Criterion of Empirical Grounding in the Sciences". In W. González (ed.) Bas van Fraassen's Approach to Representation and Models in Science. Springer Netherlands, 2014. pp. 79-100.

Weyl, H. (1927/1963): Philosophy of mathematics and natural science. New York, Atheneum. Worrall, J., (1989): “Structural Realism: The Best of Both Worlds?”. Dialectica, 43, 99-124. 